\title{
Metodología docente para la enseñanza técnica online. La experiencia Cursosagua
}

\author{
Estruch-Juan, E. *a ${ }^{\text {, del Teso, R. }}$, Gómez, E. a , Soriano, J. ${ }^{\text {a }}$ \\ aTA, Departamento de Ingeniería Hidráulica y Medio Ambiente. Universitat Politècnica de \\ València \\ *maesjua1@ita.upv.es
}

\begin{abstract}
Resumen
La enseñanza online ha llegado para quedarse. Su éxito se debe a que ha eliminado la barrera temporal y espacial, facilitando el acceso de estudiantes de todo el mundo. Sin embargo, un curso online de calidad no puede tratarse de una adaptación de una clase presencial a una clase online. Es necesario adecuar la metodología docente a este medio. Estre trabajo ilustra la metodología docente utilizada para la formación técnica online por CursosAgua, así como los resultados obtenidos.
\end{abstract}

Palabras clave: formación online, hidráulica, formación técnica, metodología docente.

\section{Introducción}

La enseñanza online es un concepto que, desde la irrupción de las nuevas tecnologías, se ha ido normalizando en la sociedad. En 2007, con la creación del primer curso MOOC (Massive Open Online Courses - Cursos Online Masivos y Abiertos), el número de estudiantes online ha ido creciendo exponencialmente (Dumbauld, 2014).

La enseñanza online ha permitido la democratización de la enseñanza puesto que ha logrado eliminar la barrera geográfica y temporal. De este modo, pueden acceder a cursos especializados un mayor número de estudiantes, tanto profesionales que buscan ampliar sus conocimientos para progresar en su carrera profesional, como estudiantes que buscan especializarse para salir al mercado laboral.

La oferta de cursos online es muy amplia, sobre todo en lo que refiere a los MOOC. Sin embargo, aunque las plataformas educativas no dejan de publicar guías de buenas prácticas para crear cursos que motiven al alumnado y ofrezcan conocimiento de calidad (MiríadaX, 
2016; Pineda, Valdivia, \& Ciraso, 2015; Universidad Carlos III de Madrid, 2016), la realidad es que las tasas de finalización de la enseñanza online, y en particular, los MOOC, son muy bajas.

Un estudio llevado a cabo por la Escuela Europea de Dirección de Empresa (EUDE) en 2014 revela que la tasa de abandono de la formación online se encuentra sobre el 35\% en másteres y posgrados, pero cuando hablamos de MOOC, el porcentaje asciende al 90\% (Carrizosa, 2014). ¿A qué se deben estas cifras? Se podrían identificar tres aspectos relevantes: la metodología utilizada en la formación online, las tasas a abonar en los cursos y las expectativas del alumnado.

La metodología docente es uno de los aspectos clave para el éxito del curso. Un formato adaptado a la formación online y atractivo, que rompa con la monotonía que supone realizar un curso online a base de vídeos o documentos pdf descargados, aumentará las posibilidades de que el estudiante finalice la formación.

Por supuesto, no toda la culpa la tiene el formato de los cursos, también está el aspecto económico. De acuerdo con Confilegal (2017), frente a una tasa de abandono de los $90 \%$ de los cursos gratuitos (MOOC), los curso de pago se sitúan en torno al $30 \%$.

Finalmente, en cuanto a las expectativas de los alumnos, se debe salvar por un lado la dificultad de que muchos de los alumnos matriculados esperan que, por el hecho de ser online, la formación sea más fácil y les suponga menos tiempo y esfuerzo. Sin embargo, esto no suele ser así, y puede ocurrir que el estudiante no encuentre el tiempo suficiente para cumplir con los compromisos que se exigen en la formación. Por otro lado, la resolución de dudas de forma rápida y efectiva es un aspecto relevante.

CursosAgua es la marca de enseñanza online del Grupo ITA (DIHMA, Universitat Politècnica de València). Cuenta con más de 10 años de experiencia online, y actualmente imparte un máster online, 4 títulos de posgrado y más de 20 cursos online de pago sobre hidráulica urbana y modelación y gestión de abastecimientos urbanos.

Este artículo presenta una propuesta de metodología docente para la enseñanza online, aplicada a formación técnica, a través de la metodología utilizada por CursosAgua en sus cursos de hidráulica. Asimismo, se presentará la figura del tutor como un actor clave para la culminación exitosa del curso por parte del alumno. 


\section{Metodología docente para la enseñanza técnica online}

\subsection{Creación de materiales para enseñanza online en asignaturas técnicas}

La creación de contenido educacional online debe de adaptarse al medio en el que será utilizado. En muchas ocasiones los cursos online son una versión en línea de contenidos presenciales. Así, es fácil, por ejemplo, encontrar cursos compuestos únicamente por largos vídeos que simulan clases presenciales o cursos formados por descargas de documentos extensos con los contenidos del temario. Es por ello que, pese a la gran oferta educativa online, no es sencillo encontrar cursos que realmente enganchen al alumno.

Uno de los factores clave para el éxito de un curso online es la pedagogía empleada y el diseño del curso (Johnson \& Aragon, 2003). El modelo pedagógico tradicional en el que el instructor proporciona grandes cantidades de información al alumno y espera que éste la procese y asimile, ha demostrado no ser el más adecuado (Reigeluth, 2016). Se ha demostrado que la asimilación de conocimiento es mayor cuando al estudiante se le proporciona la información en pequeñas dosis y cuando los contenidos se aplican a casos prácticos que guardan similitud con el día a día del alumno. La motivación del estudiante es también un aspecto que debe cuidarse, tanto a nivel de contenidos como por parte del tutor (Johnson \& Aragon, 2003).

Los contenidos de los cursos impartidos en CursosAgua tienen en cuenta todas estas consideraciones y están diseñados específicamente para impartirlos en un entorno online. El curso está formado por un camino principal, donde se encuentra todo el temario que se estima que el alumno debe poseer para alcanzar los objetivos de aprendizaje. Este camino está formado por cortos textos acompañados de imágenes y esquemas relacionadas con el contenido, que ayudan a los alumnos a una mejor comprensión del temario estudiado, tal y como muestra la Fig. 1.

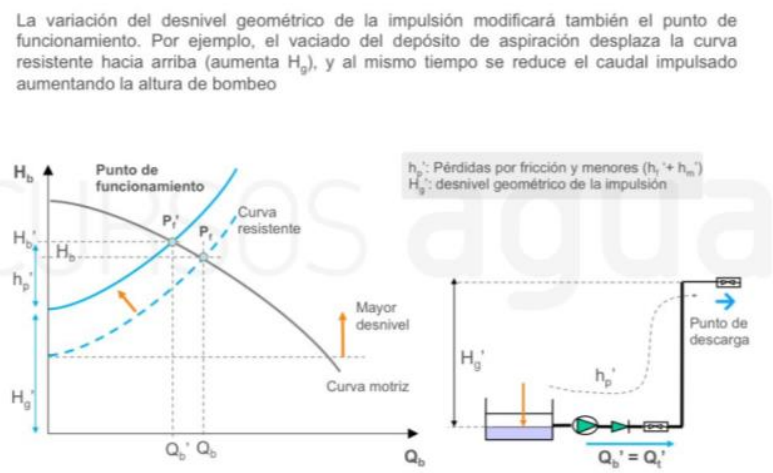

Fig. 1. Elemento del camino principal 
El camino principal cuenta también con vídeos. La función principal de éstos es explicar aquellos conceptos que son más complejos y en los cuales los alumnos presentan mayores dificultades de comprensión. Se trata de vídeos cortos, de no más de 5 minutos, puesto que se ha demostrado que la atención del alumno disminuye cuando la longitud del video es mayor (Guo, Kim, \& Rubin, 2014; Hsin, Cigas, \& Science, 2013).

La tipología de vídeos utilizada varía en función del objetivo a cumplir. Para explicar el uso de software se utilizan screencast. Los conceptos técnicos más complejos se explican con vídeos donde aparecer el profesor como protagonista, apoyado por transparencias que le permiten explicar con claridad los contenidos del vídeo. Por último, los objetivos del curso, la introducción de las unidades y los conceptos teóricos que no necesitan de explicación visual, se exponen empleando vídeos con el busto del profesor como protagonista, sin apoyo de diapositivas.

Además de los contenidos situados en el camino principal del curso, se cuenta con material adicional en forma de descarga o caminos alternativos. Las descargas pueden ser documentos de texto que proporcionen más información, enlaces a otros sitios web o casos prácticos que el alumno debe resolver. Los caminos alternativos son desvíos del camino principal que ofrecen contenido que el alumno puede elegir si desea visitar o no. Estos desvíos siguen el mismo formato que el camino principal.

Estos contenidos adicionales tienen dos objetivos. El primero de ellos es ofrecer información extra que puede ser de interés para el alumno, pero que no es necesaria para lograr los objetivos didácticos del curso. El segundo objetivo es ofrecer información sobre conceptos que son necesarios para comprender lo que se está explicando. Son conceptos que se dan por sabidos, pero que se ofrecen para ser repasados y recordados.

\subsection{Desarrollo del curso}

En muchos cursos online el temario se va desbloqueando de forma temporal, por lo que cada cierto tiempo se habilita un nuevo tema. Esta filosofía tiene algunas ventajas, ya que, se garantiza que los alumnos visualicen el contenido secuencialmente, siendo más fácil comprobar que están siguiendo el calendario del curso. Sin embargo, el mayor inconveniente de este método es que los alumnos que avanzan más rápido quedan parados hasta la nueva fecha de apertura.

En el extremo opuesto se pueden encontrar los cursos cuyo temario está completamente abierto desde un principio. Se debe tener en cuenta, que los contenidos, sobretodo en asignaturas técnicas, está ordenado de forma que se empieza desde los conocimientos más básicos para ir construyendo una base sólida que ayude al alumno a comprender los conceptos 
más complejos. En este caso, se corre el riesgo de que el alumno se salte alguna parte necesaria del temario para entender desarrollos futuros.

Los cursos ofertados por CursosAgua se encuentran disponibles en cualquier momento del año. Al tener alumnos que empiezan a lo largo de todo el año, es inviable ir abriendo los temas de acuerdo a fechas predefinidas. Para este caso, se estima más conveniente que sea el mismo alumno quien vaya avanzando a su ritmo, en función de su disponibilidad temporal, y siempre con el apoyo y seguimiento de los tutores. De este modo, los contenidos están bloqueados desde un inicio, y es el alumno quien los desbloquea conforme va desarrollando el temario.

Cabe resaltas que, aunque la formación online permite mucha flexibilidad en cuanto a horarios y fechas de realización de los cursos, es recomendable establecer una fecha de finalización para lograr que el alumno culmine con éxito la formación.

\subsection{Evaluación}

La evaluación utilizada por Cursosagua está basada en 4 elementos de evaluación (Del Teso March, Estruch Juan, Gómez Sellés, \& Soriano Olivares, 2018): puntos de control, test de las unidades, test final y entrega de ejercicios. La combinación de estos elementos conforma un sistema de evaluación continua en el que el alumno va superando pruebas en cada unidad didáctica. De esta forma, es inmediato conocer si está asimilando los conceptos correctamente y, si no es el caso, el tutor lo detecta rápidamente para remediar la situación.

Los puntos de control son el primer tipo de evaluación con la que se encuentran los alumnos. Se trata de preguntas prácticas, aplicadas a ejercicios a resolver o a conceptos teóricos clave visualizados. Se localizan a lo largo de las unidades didácticas. Los resultados obtenidos ayudan al alumno a saber si está comprendiendo el temario adecuadamente.

El test de unidades es una evaluación que se habilita una vez se ha finalizado una unidad. Su intención es evaluar si los conocimientos de la unidad se han adquirido correctamente y si los puntos de control han servido para aderezar las faltas de conocimiento.

Al finalizar cada curso el alumno debe realizar un test final en el que se evalúa si ha adquirido los objetivos de aprendizaje.

Finalmente, al tratarse de una formación técnica, la aplicación de los conceptos aprendidos a casos prácticos reales es la mejor forma de que los alumnos fijen los conocimientos adquiridos. A lo largo del curso los alumnos deben resolver ejercicios prácticos que han de enviar a los tutores para su evaluación. Una vez realizado y entregado, los tutores lo corrigen y proveen feedback en un plazo inferior a 24 horas. En el caso de que el caso no esté bien resuelto, indican al alumno en qué conceptos ha fallado para que pueda subsanarlos y volver 
a enviarlo para su evaluación. No todos los cursos tienen ejercicios, siendo una característica de los más técnicos, donde este tipo de evaluación es clave para alcanzar los objetivos del curso.

\subsection{El tutor como elemento imprescindible de la formación online}

El tutor es un elemento crucial para garantizar el éxito de un curso online (Pineda et al., 2015) ya que debe garantizar una respuesta rápida a las consultas del alumno y de forma continua. La metodología CursosAgua está completamente alineada con esta filosofía, ya que el tutor es quien realizará un seguimiento al alumno para asegurarse que el desarrollo del curso es el adecuado para superar el curso y finalizarlo dentro del plazo previsto.

Entre sus tareas están contestar las dudas de los alumnos y guiarlos en cualquier consulta que puedan tener sobre el curso o su funcionamiento. Garantizando la respuesta en un breve plazo de tiempo. Por supuesto, en muchos casos, no es una contestación inmediata, ya que cerca del $40 \%$ de los alumnos provienen de Latinoamérica. Sin embargo, las preguntas se suelen contestar en un plazo inferior a 24 horas.

El tutor es también quien corrige los ejercicios entregados por los alumnos y les proporciona la retroalimentación acerca de la idoneidad de la solución propuesta. Al tratarse de una formación ingenieril y técnica, existen multitud de soluciones posibles para los casos prácticos y, por tanto, es imprescindible la revisión de la resolución de los ejercicios planteados por los tutores. Los tutores son accesibles a los alumnos por diversos medios como son el correo interno de la plataforma CursosAgua, correo electrónico, teléfono o videoconferencia. Siendo los dos primeros los más utilizados por los alumnos.

\section{Resultados}

Los resultados obtenidos por dicha metodología docente son positivos. Las encuestas contestadas por los alumnos muestran que éstos están muy satisfechos con la formación. Las encuestas son realizadas por el Centro de Formación Permanente de la Universitat Politècnica de València, centro del que dependen los cursos ofrecidos por Cursosagua.

La Fig. 2 muestra los resultados de dicha encuesta en el Máster en Gestión Eficiente del Agua urbana. En particular, las preguntas I, J, N y O son las referidas a la metodología docente del curso, y muestran resultados excelentes, todas por encima de 9. Asimismo, las preguntas B, C y D también cuentan con buenos resultados. Estas preguntas están dirigidas a evaluar la relación del alumno con el tutor y la idoneidad de la respuesta del tutor ante las sobre cuestiones que les puedan surgir. 


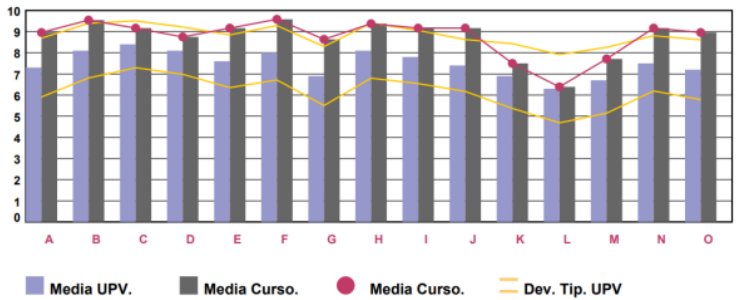

Enunciados de las preguntas:

A. La organización general del curso es buena.

B. Puedo contactar con la dirección/coordinación del curso siempre que lo solicito.

C. El trato recibido por parte del equipo de dirección/coordinación del curso es correcto

D. En términos generales, la relación profesor-alumno es buena.

E. El material de promoción consultado tenía la información suficiente.

F. El horario actual del curso se adapta a mi disponibilidad.

G. Hay suficiente coordinación entre las diferentes asignaturas.

H. Considero que la realización del curso puede ser útil en mi campo profesional.

I. En términos generales, la documentación entregada en el curso es útil para mi.

J. Los métodos de enseñanza utilizados me parecen adecuados el tipo de curso.

$K$. Las actividades complementarias (visitas a empresas, etc.), en caso de haberse organizado, me parecen interesantes.

L. La realización del curso me ayuda a crear relaciones profesionales útiles.

M. La relación entre la calidad del curso y su precio de inscripción me parece adecuada. $\quad 7,7$

$\begin{array}{ll}\text { N. Opino que la calidad del curso es buena. } & 9,2\end{array}$

O. Mis expectativas ante el curso quedan satisfechas.

Fig. 2. Resultado de las encuestas del título propio de posgrado: Máster en gestión eficiente del agua urbana

Por otro lado, acerca del porcentaje de abandono, en la última edición 17/18 ésta fue de un $19 \%$ en cursos individuales. En cuanto a los 4 títulos de posgrado que se ofertaron, la tasa de abandono fue del 28\%, llegando al 18\% en el Máster en Gestión Eficiente del Agua Urbana. Estos resultados se consideran satisfactorios ya que los porcentajes se encuentran por debajo de la tasa de abandono de este tipo de formación (35\%), aunque se está trabajando para seguir mejorando. Para ello, se están realizando mejoras en los vídeos y en el entorno educativo.

La tasa media de abandono se puede observar que es superior en los títulos de posgrado. Esto puede ser explicado por el hecho de que realizar un título de estas características, en el caso de CursosAgua, supone entre 210 y 660 horas de formación. Así, se trata de un tipo de formación que requiere más sacrificio y dedicación que realizar un único curso online. Los alumnos que realizan los posgrados de CursosAgua son en su mayoría profesionales del sector del agua, que compaginan sus estudios con sus labores profesionales. Es por ello, que no todos son capaces de dedicar todo el esfuerzo que se requiere y abandonan la formación o la posponen para finalizarla en ediciones posteriores. 


\section{Conclusión}

La metodología docente es uno de los factores clave para lograr una formación online exitosa y de calidad. Este trabajo muestra la experiencia de CursosAgua en la docencia online de formación técnica centrada en la hidráulica urbana y la gestión eficiente del agua urbana.

A lo largo del artículo se ha detallado la metodología docente utilizada, centrada en la creación de materiales adecuados para la docencia online, el formato del curso en cuanto a la visualización del temario, el método de evaluación utilizado así como el papel del tutor en la motivación y tutorización del alumno.

A continuación, se resumen los principales factores identificados como clave para garantizar una formación online de calidad y que motive al alumno. En primer lugar, se ha destacado la importancia de que los contenidos sean especialmente diseñados para ser impartidos en un medio online. Para ello, es importante que los contenidos estén presentes en distintos formatos (imagen, vídeo y texto) para romper la monotonía. Además, en un entorno online no se puede evaluar la atención del alumno. Es por ello que, para evitar distracciones y falta de atención, se propone presentar los contenidos de manera directa, clara, concisa e ilustrativa, en pequeñas dosis fácilmente digeribles por el alumno..

En segundo lugar, se recomienda que sea el alumno quien fije su ritmo de trabajo en función de su disponibilidad temporal. Sin embargo, los cursos deben tener definida una fecha de finalización para lograr que los alumnos culminen con éxito la formación.

En tercer lugar, se recomienda emplear una evaluación continua. Este tipo de evaluación ofrece grandes ventajas a la hora de detectar deficiencias en la consecución de los objetivos docentes del curso y solucionarlas rápidamente.

Finalmente, se ha destacado el papel del tutor, como elemento imprescindible en la formación online, siendo éste el encargado de fomentar la motivación de los alumnos y resolver cualquier duda o consulta que les pueda surgir. El tiempo de respuesta debe ser reducido para que los alumnos no queden parados esperando la resolución de una duda que les impide continuar con el desarrollo del curso.

Como consecuencia, los resultados obtenidos por dicha metodología docente son positivos, tal y como indican el grado de satisfacción del alumnado, así como la tasa de abandono, que se mantiene por debajo de los valores medios obtenidos por la formación online. 


\section{Referencias}

Carrizosa, S. (2014, January 12). La deserción puede con los cursos 'online.' El País. Retrieved from https://elpais.com/economia/2014/01/10/actualidad/1389360489_728192.html

Confilegal. (2017, June 14). La formación “on line" no funciona del todo bien: $30 \%$ de abandono en los cursos de pago y $90 \%$ en los gratuitos. Confilegal. Retrieved from https://confilegal.com/20170614-la-formacion-on-line-no-funciona-del-bien-30-abandono-loscursos-pago-90-los-gratuitos/

Del Teso March, R., Estruch Juan, E., Gómez Sellés, E., \& Soriano Olivares, J. (2018). Sistema de evaluación para la formación a distancia de profesionales. In INRED 2018. Congreso Nacional de Innovación Educativa y de Docencia en Red (pp. 875-888). http://doi.org/10.4995/inred2018.2018.8748

Dumbauld, B. (2014, July, 11). A brief history of online learning [Infographic]. Retrieved from https://www.straighterline.com/blog/brief-history-online-learning-infographic/. [Access 22 May 2019]

Guo, P. J., Kim, J., \& Rubin, R. (2014). How Video Production Affects Student Engagement: An Empirical Study of MOOC Videos. In L@S '14 Proceedings of the first ACM conference on Learning @ scale conference (pp. 41-50). Atlanta, Georgia, USA. http://doi.org/10.1145/2556325.2566239

Hsin, W., Cigas, J., \& Science, C. (2013). Short Videos Improve Student Learning in Online. Journal of Computing Sciences in College, 28(5), 253-259.

Johnson, S. D., \& Aragon, S. R. (2003). An instructional strategy framework for online learning environments. New Directions for Adult and Continuing Education, 2003(100), 31-43. http://doi.org/10.1002/ace.117

MiríadaX. (2016). Consejos rápidos para la creación y gestión de un MOOC ( massive open online course).

Pineda, P., Valdivia, P., \& Ciraso, A. (2015). Actividades en Moodle: Manual de buenas prácticas pedagógicas.

Retrieved

from https://ddd.uab.cat/pub/estudis/2016/149926/Moodle_buenas_practicas.pdf

Reigeluth, C. M. (2016). Teoría instruccional y tecnología para el nuevo paradigma de la educación. Revista de Educación a Distancia (RED), (50). http://doi.org/10.6018/red/50/1a

Universidad Carlos III de Madrid. (2016). Cómo crear un MOOC de éxito con Open edX. Retrieved from https://edge.edx.org/courses/course-v1:UC3Mx+UC3Mx.1x+2016_17_T2/about

Vila, R. R., Mengual-Andrés, S., \& Guerrero, C. S. (2014). Evaluación de la calidad pedagógica de los MOOC. Profesorado, 18(1), 27-41. 